\title{
Bases de datos con información estadística a escala municipal y comarcal en Internet en España
}

\section{Fernando Mikelarena Peña}

Departamento de Ciencias de la Documentación e Historia de la Ciencia Universidad de Zaragoza

\subsection{Resumen}

Se analizan las bases de datos que en España disponen de información estadística a nivel municipal y comarcal, y que tienen acceso a través de Internet. Para ello, se analizan las bases de datos y la composición de los registros que ofrecen los servicios de Estadística de Murcia, de Castilla y León, Aragón, Canarias, Andalucía, Cataluña, Comunidad de Madrid y el País Vasco.

Palabras clave: Información estadística. Bases de datos on-line. Municipios. Comarcas.

\subsection{Abstract}

Internet-available statistical databaseson Spain at the municipality and local level are analyzed. The structure and services of the statistical databases of the autonomous regions of Murcia, Castilla y León, Aragón, Canarias, Andalucía, Cataluña, Madrid and País Vasco are described.

Keywords: Statistical information. On-line databases. Municipalities. Regions.

\section{Introducción}

Los principales productores y difusores de información estadística en la España actual son el Instituto Nacional de Estadística y los organismos estadísticos autonómicos, éstos últimos ciertamente ya consolidados en la mayor parte de las comunidades autónomas.

El Instituto Nacional de Estadística es el organismo estadístico por excelencia de la Administración Central del Estado. Surgió en 1945, habiendo tenido diversos antecesores desde mediados del siglo XIX: la Comisión General de la Estadística General del Reino, el Instituto Geográfico y Estadístico, la Dirección General de Estadística, etc. 


\section{6}

El origen legal de los organismos estadísticos autonómicos parte del hecho de que en el artículo 149.3 de la Constitución de 1978 se indicaba que la estadística para fines no estatales podía ser una competencia que podría encuadrarse dentro de "las materias no atribuidas expresamente al Estado", pudiendo corresponder a las Comunidades Autónomas en el caso de que así lo establecieran los respectivos estatutos de autonomía de cada comunidad. Con arreglo a esas posibilidades que brindaba el texto constitucional, la mayoría de las Comunidades Autónomas recogieron en sus estatutos (o norma equivalente, como el Amejoramiento del Fuero, en el caso navarro), la competencia en materia estadística para fines no estatales, aunque con distintos grados de asunción. Con todo, la entrada en vigor tanto de la Ley Orgánica 9/92 de ampliación de competencias a las Comunidades Autónomas que accedieron a la autonomía por el artículo 143 de la Constitución como de la subsiguiente reforma de estatutos en dichas Comunidades hizo que todas ellas sin excepción cuenten ahora con plena competencia exclusiva en materia de estadística para fines propios (Menéndez Bañuelos, 1994, p. 13-15).

Ahora bien, además de la razón legal, en el surgimiento de los organismos estadísticos autonómicos también intervino una motivación funcional. Considerando la trascendencia de la información estadística como instrumento que permite conocer la realidad demográfica, económica y social de la región, conocimiento que constituía un requisito necesario para la toma de decisiones por parte de cada administración autonómica, aquella motivación funcional se refería a la circunstancia de que dichas administraciones necesitan "disponer de información regional, provincial, comarcal, municipal y de unidades territoriales menores", información que, sobre todo en relación con los niveles de desagregación espacial infraprovincial, no facilita en INE más que excepcionalmente (Orensanz y otros, 1995, p.66).

Hoy en día existen organismos estadísticos autonómicos en la mayor parte de las Comunidades Autónomas españolas. Algunos de ellos (como los del País Vasco, Cataluña, Galicia, Andalucía, Valencia, Canarias, Madrid, Navarra, Baleares y Aragón) tienen el rango de Institutos Regionales de Estadística. Los organismos de estadísticos de Castilla y León, Murcia y Cantabria no tienen la categoría de Instituto dentro del organigrama autonómico, sino la de Dirección General en los dos primeros casos y de Servicio en el tercero. En las demás comunidades existen servicios no explícitamente de Estadística, pero que incorporan funciones de génesis y de distribución de información estadística, tanto en soporte de papel como por Internet. Es el caso del Servicio de Planificación, Estadística y Análisis Económico de la Junta de Extremadura. 
En lo que resta de artículo, nos centraremos en el análisis de las bases de datos con información estadística a escala municipal y comarcal existentes en España que se difunden libremente a través de Internet.

\section{Bases de datos con información estadística a escala municipal y comarcal en Internet en España}

Todas las bases de datos que difunden a través de Internet información estadística desagregada hasta el plano municipal y comarcal existentes en España son producidas y difundidas por organismos estadísticos autonómicos. Hay que subrayar el hecho de que el surgimiento de dichas bases responde a un afán por parte de las administraciones autonómicas de poner a disposición, tanto del público especializado (investigadores, gabinetes de estudios, medios de comunicación) como del general, información estadística en principio encaminada hacia la propia administración. Ese afán, común por otra parte a los organismos estadísticos del mundo occidental, está ocasionado en última instancia por un cambio, constatado en los últimos decenios, en la noción de relación entre administrador y administrado por el que se considera que la Administración Pública dispensa servicios cuyo cliente es el ciudadano, haciéndolo además de forma receptiva (Organización de Cooperación y Desarrollo Económico, 1987, 39-41).

Las bases de datos con información estadística a escala municipal y comarcal existentes en España que se difunden libremente a través de Internet se localizan en los servidores del Instituto Vasco de Estadística (EUSTAT), del Instituto Canario de Estadística (ISTAC), del Instituto de Estadística de Andalucía (IEA), del Instituto de Estadística de Cataluña (IDESCAT), del Instituto de Estadística de la Comunidad de Madrid, de la Dirección General de Estadística de Castilla y León, del Instituto Aragonés de Estadística (IAE), del Centro Regional de Estadística de Murcia y del Servicio de Planificación, Estadística y Análisis Económico de la Junta de Extremadura.

\subsection{País Vasco}

El Instituto Vasco de Estadística (EUSTAT) pone a disposición del público en la dirección http://www.eustat.es/spanish/estad/municipal.html un amplio elenco de indicadores relativos a cada municipio y a cada comarca, siendo posible posteriormente tanto extraer informaciones para cada territorio histórico como llevar a cabo comparaciones entre una pareja de municipios o de comarcas. Las informaciones que hay para cada municipio y para cada comarca son las siguientes:

1) Número de nacidos vivos por sexo, orden de nacimiento y estados civil de las madres en 1998. 
2) Población de derecho según sexo y tramo de edad y densidad de población en 1996.

3) Población total y por sexos, movimiento natural y movimientos migratorios en 1997.

4) Estado civil anterior de los casados en 1998.

5) Número de defunciones totales, por sexo y por causa de muerte y crecimiento vegetativo en 1998.

6) Distribución de la población según personas sedentarias, migrantes externos e inmigrantes en 1996.

7) Movimientos migratorios según su clase en 1997.

8) Tamaño medio de las unidades familiares y distribución del número medio de integrantes según edad y su situación en relación con el mercado de trabajo en 1996.

9) Núcleos familiares según el tipo de núcleo en 1996.

10) Número y distribución de los centros extrahospitalarios en 1998.

11) Número de alumnos en cada nivel de enseñanza preuniversitaria según la titularidad del centro en el curso 1998/1999.

12) Número de alumnos en cada nivel de enseñanza preuniversitaria según el modelo linguí́stico en el curso 1998/1999.

13) Distribución de la población de más de 10 años según nivel de instrucción en 1996.

14) Distribución de la población de 2 y más años según el conocimiento de la lengua vasca en 1996.

15) Distribución de la población de 2 y más años según la lengua materna y la lengua hablada en casa en 1996.

16) Distribución de la población de 5 años o más según su movilidad lingüística en 1996.

17) Distribución de la población de 5 años o más según la lengua de uso en casa en 1996.

18) Número de centros asistenciales según tipo de centro en 1998.

19) Número de establecimientos según rama de actividad en 1999.

20) Distribución de las viviendas según su clase en 1996.

21) Distribución de las viviendas principales según sus instalaciones y servicios en 1996.

22) Distribución de los edificios según su estado. 
23) Distribución de los edificios normales según su destino.

24) Distribución de los edificios normales según los equipamientos del entorno.

25) Distribución de los edificios normales según su número de plantas y de huecos.

26) Resultados de las elecciones autonómicas de 1998.

27) Resultados de las elecciones municipales de 1995.

28) Distribución de la población según su actividad en 1996.

29) Distribución de la población ocupada según ramas de actividad en 1996.

30) Paro registrado en el INEM en 1999 y 2000.

31) Estructura del valor añadido municipal por sectores económicos.

32) PIB municipal per cápita en 1996.

33) Liquidación de los ingresos y gastos municipales en 1998.

34) Explotaciones agrarias censadas y superficie agraria total en 1989 y en 1989.

35) Distribución de los usos del suelo en 1996.

36) Distribución de la superficie forestal arbolada por especies en 1996.

37) Distribución de la superficie y distribución de las masas de cultivo en 1999.

38) Régimen de tenencia de la tierra en 1999.

39) Personalidad jurídica de los propietarios de tierra en 1999.

40) Distribución de la cabaña ganadera en 1999.

41) Distribución de los trabajadores en las explotaciones agrarias en 1999.

42) Distribución del parque de vehículos en 1998.

43) Número de líneas telefónicas y tasa por mil habitantes.

44) Número y distribución de las entidades bancarias en 1998.

45) Extensión y altitud del municipio.

\subsection{Andalucía}

El Instituto de Estadística de Andalucía (IEA) integra en su página web el SIMA (Sistema de Información Multiterritorial de Andalucía) que ofrece abundante información estadística sobre los municipios y las provincias de la Comunidad en la dirección http://www.iea.junta-andalucia.es/sima/ . Los aspectos que recoge esta base de datos relativos a información estadística municipal son los siguientes: 
1) Territorio: características geográficas (extensión superficial, longitud y latitud y altitud sobre el nivel del mar), situación (distancia a las capitales) y características del suelo (suelos según litología, pendiente, erosión $\mathrm{y}$ formas de uso).

2) Población: distribución y crecimiento de la población (población de derecho total y población de derecho por sexo), estructura de la población (población de derecho por grupos de edad y sexo, población de más de diez años por nivel de instrucción, población según nacionalidad y sexo, población según la relación entre el lugar de nacimiento y el de residencia por sexo y tasa de escolaridad por grupos de edad), movimiento natural de la población (nacidos vivos por residencia materna y sexo, matrimonios por lugar donde fijan su residencia, defunciones por lugar de residencia y sexo y crecimiento vegetativo) y migraciones (emigración total, emigración por sexo, inmigración total e inmigración por sexo).

3) Sociedad: enseñanza y formación (centros públicos y privados por niveles educativos, centros de adultos públicos y privados, alumnos en centros públicos y privados por nivel educativo, alumnos en centros de adultos públicos y privados, profesores en centros públicos y privados por nivel educativo, profesores en centros de adultos públicos y privados), recursos sanitarios y salud de la población (recursos de atención primaria, recursos de atención especializada, farmacias, defunciones por causa de muerte), edificios y viviendas (edificios por destino, edificios por instalaciones y servicios, viviendas según su clase, viviendas de nueva construcción y viviendas de rehabilitación protegida), elecciones (datos sobre el censo electoral, el número de votantes, los votos, la abstención y los votos de las candidaturas tanto en las elecciones generales como en las autonómicas y municipales), cultura y tiempo libre (número de bibliotecas públicas locales y número de prestamos, de consultas y de lectores en ellas y número de cines y de pantallas de cine y aforo en salas de cine) y servicios sociales y protección social (pensiones no contributivas de la Seguridad Social e importe de las mismas).

4) Economía: sector agrario (distribución de la tierra por aprovechamientos, superficie de los diferentes tipos de cultivos, superficie de las explotaciones agrarias por aprovechamiento y por régimen de tenencia, explotaciones agrarias por tamaño de explotación y por soporte territorial, máquinas agrícolas por tipo, titulares de explotaciones agrícolas por edad, empresarios por ocupación principal, unidades de trabajo en cada año en explotaciones agrarias y unidades ganaderas por tipos), sector industrial (inversiones en industria, en construcción en la agricultura y 
en servicios en el Registro Industrial y consumo de energía eléctrica), turismo (establecimientos hoteleros según clase y categoría, plazas en establecimientos hoteleros por clase y categoría, número de apartamentos por categoría, número de plazas en apartamentos por categorías, número de restaurantes y cafeterías por categorías, número de plazas en restaurantes y cafeterías por categorías, número de campings por categorías y número de plazas en campings por categorías), transporte y comunicaciones (parque de vehículos por tipo y servicio, parque de camiones por carga, autorizaciones de transporte según tipo y por carga y por servicio, número de taxis, líneas telefónicas en servicio), actividad financiera y empresarial (oficinas bancarias por tipo de entidad; número de establecimientos por actividad económica, forma jurídica y tramo de empleo, número de cooperativas por actividad económica y clase, número de socios de cooperativas por actividad económica y clase, número de trabajadores de cooperativas por actividad económica y clase, número de sociedades anónimas laborales por actividad económica, número de socios de sociedades anónimas laborales por actividad económica y número de trabajadores de sociedades anónimas laborales por actividad económica), locales (locales censados por categorías y sectores de actividad y personas ocupadas en locales y por sector de actividad), macromagnitudes (renta familiar disponible por habitante).

5) Mercado de trabajo: población activa (población activa por sexo y tasa de actividad por sexo), población ocupada (población ocupada por sexo, sector económico y situación profesional), población desempleada (población parada por sexo, paro registrado a 31 de marzo, medias anuales de paro registrado por sexo, grupo de edad, nivel de estudios, grupo de ocupación solicitada y sectores de actividad).

6) Hacienda: Impuestos de Bienes Inmuebles (número de recibos, valor catastral, base imponible y cuota del Impuesto de Bienes Inmuebles de naturaleza urbana; titulares catastrales, valor catastral, base imponible, cuota y superficie imponible del Impuesto de Bienes Inmuebles de naturaleza rústica), Impuesto de Actividades Económicas empresariales, profesionales y artísticas por divisiones; e Impuesto sobre la Renta de las Personas Físicas (número de declaraciones, rentas netas declaradas y renta neta declarada por tipo de rendimiento).

\subsection{Canarias}

El Instituto Canario de Estadística difunde en Internet la Base de Datos Insular y municipal en la dirección http://www.istac.rcanaria.es/ . En ella conta- 
mos para cada municipio de la comunidad canaria y para cada isla con la siguiente información:

a) Territorio y medio ambiente: altitud de la capital municipal, altitud máxima, altitud mínima, coordenadas U.T.M., latitud, longitud, distancia a la capital, perímetro municipal y superficie.

b) Población: densidad, población de derecho, crecimiento vegetativo, defunciones, matrimonios y nacimientos para el periodo 1975-1999.

c) Trabajo: empleos por cuenta ajena según sección, empleos por cuenta propia según sección, total de empleos según sección, paro registrado y número de prestaciones e importe de prestaciones por favor familiar, invalidez, jubilación, orfandad y viudedad para el periodo 1986-2000.

d) Educación, formación e investigación: número de alumnos de los diferentes niveles educativos, centros de enseñanza reglada y profesores de enseñanza reglada para el periodo 1988-1998.

e) Justicia y participación social: censo electoral, número de votantes, votos blancos, votos nulos, votos válidos, abstenciones y votos de cada candidatura en las diferentes convocatorias electorales del periodo $1993-$ 2000.

f) Urbanismo, vivienda y construcción: cédulas de habitabilidad, viviendas de promoción libre iniciadas, viviendas de promoción libre terminadas, viviendas de protección oficial de promoción privada iniciadas, viviendas de protección oficial de promoción privada terminadas, viviendas de protección oficial de promoción pública iniciadas y viviendas de protección oficial de promoción pública terminadas para el periodo 1987-1998.

g) Sector primario: cabezas de ganado de las diversas especies; equipos de recolección, de siembra, de abonado y de trabajo del suelo; superficies de los diversos cultivos, motores, otros equipos y tractores para el periodo 1986-1999.

h) Sector secundario: abonados a UNELCO y consumo de energía eléctrica para el periodo 1985-1999.

i) Sector servicios: altas del Impuesto de Actividades Económicas, oficinas bancarias y distribución, bares, cafeterías, restaurantes, número de establecimientos extrahosteleros y plazas en ellos, número de establecimientos hoteleros y plazas en ellos y número de vehículos y distribución por tipos para el periodo 1985-1999.

j) Sector público: D.R.N. de los ayuntamientos, O.R.N. de los ayuntamientos, declarantes del IRPF, recibos del Impuesto de Bienes Inmuebles urbanos, rendimientos del IRPF, superficie imponible, titulares y valor 
catastral del Impuesto de Bienes Inmuebles rústicos para el periodo 1990-1998.

\subsection{Castilla y León}

La Dirección General de Estadística de la Consejería de Economía y Hacienda de la Junta de Castilla y León cuenta en la dirección http://www.jcyl.es/jcyl/ceh/dge/municipios con la base denominada "Datos Municipales de Castilla Y León" en la que figuran para cada municipio los siguientes datos:

a) Datos geográficos: código del municipio; distancia a la capital provincial y número de la hoja del Mapa Topográfico Nacional donde se localiza el municipio.

b) Población: distribución de la población de hombres y mujeres por intervalos de edad en 1991 y 1996, movimiento natural de la población entre 1990 y 1996, distribución de la población por nivel de instrucción en 1991, distribución de la población activa masculina y femenina en 1991 y población de hombres y mujeres por clases pasivas en 1991.

c) Educación: datos de educación no universitaria del curso 1997-1998.

d) Economía: distribución del paro registrado clasificado por sexo, edades y nivel de estudios en 1998; parque de vehículos en 1998; distribución de las viviendas familiares en principales, secundarias o desocupadas en 1991; número de líneas telefónicas en 1997; número de entidades financieras en 1998; distribución de la superficie agraria por tipos de cultivo en 1996; porcentajes de declarantes, base imponible y rendimiento medio declarado en el Impuesto sobre la Renta de las Personas Físicas en 1997; y datos sobre el sector de la hostelería en 1998.

e) Elecciones: Datos de las elecciones municipales y autonómicas de 1995 y de las generales de 1996.

Asimismo, existe una Biblioteca de Mapas de Datos Municipales de Castilla y León en la dirección http://www.jcyl.es/jcyl/ceh/dge/municipios/mapas.htm en la que se pueden ver gráficamente aspectos como la densidad de población en 1996, la variación de la población entre 1991 y 1996, diversos índices de dependencia y de envejecimiento de la población en 1991 y el uso predominante de las tierras en 1996 


\subsection{Cataluña}

El Instituto de Estadística de Cataluña (IDESCAT) presenta para cada municipio catalán en la dirección http://www.idescat.es/estbasiq/municipi/ municipi.stm los siguientes datos:

a) Datos geográficos: superficie y altitud de cada municipio y comarca en la que se encuentra.

b) Población: distribución de la población en grupos de edades en 1981, 1991 y 1996 y comparación con los datos de la comarca respectiva y de Cataluña; distribución de la población por lugar de nacimiento en 1991 y 1996 y comparación con los datos de la comarca respectiva y de Cataluña; número de nacimientos, defunciones, matrimonios y crecimiento natural en 1991 y 1998 y comparación con los datos de la comarca respectiva y de Cataluña; distribución de los hogares según el número de personas que los integran e 1991 y 1996 y comparación con los datos de la comarca respectiva y de Cataluña; distribución de la población de más de diez años según su nivel de instrucción en 1991 y 1996 y comparación con los datos de la comarca respectiva y de Cataluña; distribución de la población catalanoparlante en 1981, 1991 y 1996 y comparación con los datos de la comarca respectiva y de Cataluña.

c) Edificios y viviendas: distribución de los edificios según su grado de conservación en 1980 y 1990 y comparación con los datos de la comarca respectiva y de Cataluña; distribución de las viviendas familiares según su tipología en 1981 y 1991 y comparación con los datos de la comarca respectiva y de Cataluña; distribución de las viviendas principales según su superficie útil en 1991 y comparación con los datos de la comarca respectiva y de Cataluña; y distribución de las viviendas principales según sus instalaciones y servicios en 1991 y comparación con los datos de la comarca respectiva y de Cataluña.

d) Agricultura: distribución de la superficie agraria en 1989 y comparación con los datos de la comarca respectiva y de Cataluña.

e) Economía: distribución de la población ocupada residente por sectores de actividad en 1986, 1991 y 1996 y comparación con los datos de la comarca respectiva y de Cataluña; distribución de los establecimientos y profesionales por grandes sectores de actividad económica en 1995 y 1998 y comparación con los datos de la comarca respectiva y de Cataluña; distribución de las empresas industriales por sectores de actividad en 1995 y 1998 y comparación con los datos de la comarca respectiva y de Cataluña; distribución de las empresas de comercio minorista en 1995 y 1998 y comparación con los datos de la comarca 
respectiva y de Cataluña; distribución de las empresas de servicios en 1995 y 1998 y comparación con los datos de la comarca respectiva y de Cataluña; número de líneas telefónicas en servicio en 1993 y 1997 y comparación con los datos de la comarca respectiva y de Cataluña; distribución del parque de vehículos en 1991 y 1998 y comparación con los datos de la comarca respectiva y de Cataluña; distribución de las entidades financieras en 1986 y 1997 y comparación con los datos de la comarca respectiva y de Cataluña; número de contribuyentes al impuesto sobre bienes inmuebles urbanos al impuesto sobre bienes inmuebles rústicos y recaudación en 1991 y 1999 y comparación con los datos de la comarca respectiva y de Cataluña; distribución del impuesto sobre la renta de las personas físicas y del impuesto sobre el patrimonio de las personas físicas en 1986 y 1997 y comparación con los datos de la comarca respectiva y de Cataluña.

Esa misma información existe también para cada comarca catalana en la misma dirección, añadiéndose además el montante y la distribución del producto interior bruto por sectores económicos a precios de mercado y el montante total y por individuos de la de la renta bruta familiar disponible en 1991, así como la distribución de la misma por recursos principales, en todos los casos con comparación con los datos de la comarca respectiva y de Cataluña.

Por otra parte, en otro sector de la misma página web del Instituto de Estadística de Cataluña (concretamente en la dirección http://www.idescat.es/basdades/bemc/bemc.stm ) se puede consultar el Banc d'estadistiques municipals i comarcals de Catalunya en el que hay tablas sobre los siguientes temas:

a) Estadísticas de población: estructura de la población, estimaciones de la población, fecundidad de la población femenina, movilidad espacial de la población, hogares y familias, flujos de población, proyecciones de población y población estacional.

b) Estadísticas sociales: viviendas y edificios, equipamientos y servicios, bienestar social y elecciones.

c) Estadísticas económicas: macromagnitudes; censo agrario; empresas, profesionales, establecimientos y superficies; hacienda pública: mercado de trabajo; e indicadores de actividad.

d) Otras estadísticas: indicadores geográficos.

\subsection{Aragón}

El Instituto Aragonés de Estadística (IAE) cuenta con informaciones para cada municipio y cada comarca de la comunidad autónoma sobre los siguientes 
aspectos en formato excel y/o PDF en la dirección http://www.aragob.es/eco/estadistica/espanol/iaeind02.htm :

a) Datos de la actividad económica según el Impuesto de Actividades Económicas.

b) Población: de 1991 fichas municipales del censo de ese año, población de derecho según estado civil y sexo en 1991, población de derecho según sexo y grupos de edad, población de derecho de 10 a o más años según nivel de estudios, población de derecho ocupada por sectores de actividad; datos del padrón municipal revisado para 1996, 1998 y 1999; nacimientos y defunciones para 1980-1997; y movimientos migratorios por sexo para 1988-1995.

c) Agricultura: distribución de masas de cultivo en 1999.

d) Vivienda: para 1991 viviendas familiares según año de construcción, clase, instalaciones y servicios, régimen de tenencia y superficie útil.

e) Locales: para 1991 distribución de los locales activos y auxiliares y de las personas ocupadas en ellos por nivel de empleo y tipo de local.

f) Edificios: para 1991 edificios según número de plantas, clase de propietarios y número de plantas y edificios destinados a vivienda familiar según estado, instalaciones, año de terminación y número de viviendas.

\subsection{Murcia}

El Centro Regional de Estadística de la Región de Murcia (CREM) tiene en la dirección de Internet http://www.carm.es/econet los siguientes contenidos informativos sobre los municipios de la región en formato excel:

a) Agricultura, ganadería y pesca: distribución de masas de cultivo en 1999 y en 1991-1999, maquinaria agrícola en 1999, número de cabezas de ganado por especies en 1999, pesca desembarcada por especies en 1995-1999, embarcaciones pesqueras por modalidad de pesca en 19921999, trabajadores de la pesca en 1992-1999 y empresarios agrícolas en 1989 por edades y por ocupación principal.

b) Vivienda: para 1991 viviendas según clase y viviendas principales según año de construcción, régimen de tenencia, superficie útil, número de habitaciones, instalaciones y régimen legal de la construcción; para 1999 edificios y superficies a construir de nueva planta, número de obras de rehabilitación y demolición, número medio de viviendas por edificios y licencias concedidas por tipo de obra; y para 1992-1999 evolución de las viviendas a construir y de las viviendas protegidas y libres. 
c) Información catastral: para 1999 parcelas catastrales por tipo, solares según superficie, parcelas edificadas según superficie, superficie del suelo según tipo de parcela, catastro urbano, catastro rústico e Impuesto de Bienes Inmuebles referido a los bienes de naturaleza urbana y de naturaleza rústica.

d) Movimiento natural de la población: nacimientos (evolución de nacidos vivos entre 1975 y 2000; nacidos vivos por meses en 1999 y 2000; nacidos vivos por edad de la madre en 1999 y nacidos vivos por nacionalidad de la madre en 2000), defunciones (evolución entre 1996 y 2000 y para 1998 y 2000 número según lugar de inscripción y sexo; según lugar de residencia y sexo; según edad, sexo y estado civil; de menores de un año; según profesión, y según causas de muerte) y matrimonios (evolución de los matrimonios según municipio de futura residencia en 1976-2000).

e) Población: evolución de la población de derecho por sexo en 1900 y 1991 y 1991-1999; evolución de la densidad de población en 1990-1999; población según la edad y sexo en 1996 y 1998; población según sexo y nacionalidad en 1998; población según Comunidad Autónoma de nacimiento en 1998; población extranjera según país de nacimiento en 1998; indicadores de estructura por edades en 1996; hogares según tamaño en 1991 y 1998; hogares según tipo en 1991 y 1998; hogares según presencia de ancianos y de jóvenes en 1998.

f) Servicios: número de oficinas bancarias en 1999.

g) Hacienda pública: distribución por clases de los ingresos y de los gastos municipales en 1999 y evolución de los presupuestos municipales en 1992-1999.

h) Fiscalidad: número de contribuyentes y base imponible media del IRPF en 1997-1998; rendimiento medio del IRPF según tipo en 1997-1998, y declarantes del IVA y distribución por municipios.

i) Inversión industrial: inversión industrial por destino de la inversión en 1999 e inversión industrial por sectores en ese mismo año.

j) Indicadores económicos: renta familiar bruta y renta familiar bruta disponible per cápita en 1996.

k) Movimientos migratorios: para 1996 y 1998 evolución de las inmigraciones y de las emigraciones totales según municipio de destino; evolución de los saldos migratorios totales; evolución de las migraciones interiores según municipio de procedencia y de destino, evolución de las inmigraciones de otras CCAA según municipios de destino y evolución de las inmigraciones del extranjero según municipios de destino. 
1) Movimiento laboral registrado: evolución del paro en 1992-2000; y para marzo de 2001 paro por grupos de edad y sexo, por sectores económicos, por niveles académicos y por grupos profesionales.

m) Territorio: superficie y distancia a la capital y número de incendios forestales.

n) Turismo: para el año 2000 oferta hotelera por categorías y oferta de apartamentos turísticos, de casas rurales, de campamentos turísticos y de restaurantes y cafeterías.

\subsection{Extremadura}

El Servicio de Planificación, Estadística y Análisis Económico de la Junta de Extremadura difunde en la dirección <http:www.junta.ex/consejerias/eic/ dgpp/mun2000/home.html> una base de datos, que en rigor es una adecuación de un documento PDF, en el que se recoge para cada municipio los siguientes indicadores:

1) Superficie, distancia a la capital provincial, población y densidad.

2) Porcentajes de población joven, adulta y anciana, expresados gráficamente.

3) Número de nacimientos, tasa bruta de natalidad, tasa de fecundidad y proporción niños/mujeres en edad fértil.

4) Defunciones y tasa bruta de mortalidad.

5) Distribución de la población de 10 ó más años, según su nivel de instrucción.

6) Renta familiar disponible por habitante.

7) Número de oficinas bancarias, de vehículos según su clase y de líneas telefónicas.

8) Población activa e inactiva según su relación con la actividad y distribución de los parados por sectores económicos, por grupos profesionales y por sexo y edad.

9) Superficie total y superficie cultivada.

10) Indice turístico, según la cuota del Impuesto de Actividades Económicas pagado por los establecimientos turísticos.

11) Actividades comerciales e industriales según el Impuesto de Actividades Económicas.

12) Indice de Actividad Económica según el porcentaje que suponga el Impuesto de Actividades Económicas de las actividades empresariales y 
profesionales de cada municipio en relación con el total del Impuesto de Actividades Económicas de esas actividades en la provincia respectiva.

13) Número de empresas por sectores.

\subsection{Comunidad de Madrid}

Para finalizar, el Instituto de Estadística de la Comunidad de Madrid cuenta con una base de datos denominada Almudena con muchísimos datos sobre municipios en la dirección http://www1.comadrid.es/destadis/simbad/cgi-bin/simbad.exe . Los apartados y aspectos para los que existe información para cada municipio de la comunidad autónoma son los siguientes:

a) Condiciones ambientales: territorio y climatología (altitud, distancia a la capital y extensión) y contaminación y tratamiento de residuos (residuos sólidos urbanos eliminados en vertederos controlados y residuos sólidos urbanos tratados en estación de transferencia)

b) Población y hogares: estructura y evolución (densidad de población, distribución de hogares según el número de miembros que los integran, población de derecho total y por sexos, rectificación de las cifras de la población de derecho del padrón), movimiento natural de la población (defunciones de residentes y nacimientos de madres residentes), nupcialidad (matrimonios que han fijado su residencia) y migraciones (altas y bajas totales y por sexos en la estadística de variaciones residenciales; saldo migratorio total y por sexos; emigración a otras comunidades autónomas total, por sexos y por grupos de edades; inmigración desde a otras comunidades autónomas total, por sexos y por grupos de edades).

c) Mercado de trabajo: gestión del empleo (paro registrado).

d) Cultura y ocio: actividades culturales (recaudación total y total de espectadores en salas de cine de películas españolas y de películas extranjeras, número de salas y de pantallas de cine, recaudación total de salas de cine) y actividades deportivas (número total y por sexos de participantes en la campaña de deporte infantil de aprendizaje de natación; número total y por sexos de deportistas participantes en deporte infantil).

e) Relaciones y participación social: ejercicio de los derechos fundamentales (número de electores, de abstenciones, de votantes, de votos en blanco, de votos válidos y porcentajes de votos de las distintas candidaturas en las elecciones generales, autonómicas, europeas y municipales)

f) Transportes y comunicaciones: transporte por carretera (cifras de autobuses de viajeros de transporte por carretera de servicio privado, de servicio público discrecional y de servicio público regular; cifras de vehículos de transporte de mercancías; cifras de taxis, ambulancias y 
vehículos de alquiler de transporte de viajeros por carretera), transporte urbano (cifras de estaciones de tren de cercanías; cifras de marquesinas, paradas de autobús y puntos de venta de abonos y cifras de líneas de autobuses de transporte público según su tipo) y comunicaciones (cifras de líneas telefónicas)

g) Urbanismo, vivienda y construcción: usos del suelo y planeamiento (suelo clasificado para sistemas generales, suelo clasificado como suelo especial protegido, suelo clasificado como suelo no urbanizable, suelo clasificado como suelo urbanizable, suelo clasificado como suelo urbano, distribución del suelo urbanizable según su uso y distribución del suelo urbano según su uso), catastro inmobiliario (año de revisión del valor catastral urbano y rústico, número de parcelas catastrales urbanas edificadas y distribución según su tipo, número de parcelas urbanas edificadas y distribución según su superficie, titulares de inmuebles rústicos exentos de tributar, subparcelas rústicas tributables y exentas de tributación, titulares y contribuyentes de inmuebles rústicos, parcelas rústicas y titulares de inmuebles rústicos, número de solares urbanos y distribución según su superficie, total de superficie de suelo urbano, superficie de parcelas urbanas edificadas y de solares urbanos, unidades urbanas, valores catastrales por unidad urbana, valores catastrales rústicos y valores catastrales urbanos) y vivienda y edificación (cifras de licencias de obras y distribución según finalidad de la obra, superficie a construir de edificios residenciales y no residenciales, edificios residenciales y no residenciales a construir, superficie total a construir, total de viviendas a construir, cifras de viviendas familiares y distribución por tipos).

h) Industria y energía: encuestas industriales (costes de personal, densidad industrial, producción industrial y valor añadido bruto de la industria manufacturera) y producción de la energía (energía eléctrica facturada).

i) Magnitudes económicas: contabilidad regional (renta familiar disponible per cápita y producto municipal bruto per cápita), finanzas y seguros (número de oficinas bancarias y distribución por tipos) y otros indicadores agregados (número total de altas en el Impuesto de Actividades Económicas y distribución de las mismas en los distintos sectores económicos).

j) Sector Público: presupuestos (gastos consolidados en obligaciones reconocidas de operaciones corrientes y de operaciones de capital de los presupuestos municipales liquidados, ingresos consolidados por derechos reconocidos de operaciones corrientes y de operaciones de capital de los presupuestos municipales liquidados, gastos consolidados en obligacio- 
nes y en pagos reconocidos de los presupuestos municipales liquidados e ingresos consolidados en derechos reconocidos y por recaudación líquida de los presupuestos municipales liquidados) y recaudación (recaudación del Impuesto de Actividades Económicas, del Impuesto de Bienes Inmuebles de naturaleza rústica del Impuesto de Bienes Inmuebles de naturaleza urbana; base imponible de contribuyentes, base imponible de exentos, base imponible total, cuota y deuda tributaria del Impuesto de Bienes Inmuebles de naturaleza rústica; base imponible, base liquidable, cuota y deuda tributaria del Impuesto de Bienes Inmuebles de naturaleza urbana; tipos de gravamen del Impuesto de Vehículos de Turismo según su potencia; base imponible, cuota líquida, declarantes, rendimiento medio de las rentas agrícolas, empresariales, profesionales y del trabajo del Impuesto sobre la Renta de las Personas Físicas; tipos de gravamen del Impuesto Municipal sobre Construcciones e Instalaciones y Obras y del Impuesto Municipal de Incremento del Valor de Terrenos de naturaleza urbana).

k) Directorios: total (total de ocupados y total de unidades locales), industria (establecimientos activos y unidades locales del sector industrial, total de personas ocupadas en el sector industrial y distribución por subsectores), comercio al por mayor e intermediarios de comercio (establecimientos activos, personas ocupadas y unidades locales del comercio al por mayor e intermedios de comercio), comercio al por menor (ocupados y unidades locales en el sector del comercio al por menor), servicios a empresas (establecimientos activos, personas ocupadas y unidades locales del sector servicios a empresas), talleres artesanos (número de talleres artesanos) y talleres de reparación de vehículos (establecimientos activos y personas ocupadas en el sector de talleres de reparación de vehículos).

\section{Bibliografía}

Centro Regional de Estadística de la Región de Murcia (CREM). $\mathrm{URL}=\langle\mathrm{http}: / / \mathrm{www}$. carm.es/econet $\rangle$.

Dirección General de Estadística de la Consejería de Economía y Hacienda de la Junta de Castilla y León. Datos Municipales de Castilla Y León. URL=<http://www.jcyl.es/jcyl/ceh/dge/municipios $>$.

Instituto Aragonés de Estadística

(IAE).

URL $=<$ http://www.aragob.es/eco/estadistica/espanol/iaeind02.htm $>$.

Instituto Canario de Estadística. Base de Datos Insular y municipal. $\mathrm{URL}=\langle\mathrm{http}: / / \mathrm{www}$.istac.rcanaria.es $\rangle$.

Instituto de Estadística de Andalucía (IEA). Sistema de Información Multiterritorial de Andalucía (SIMA). URL=<http://www.iea.junta-andalucia.es/sima $>$.

Scire. $6: 1$ (en.-jun. 2000) 125-142 


\section{Fernando Mikelarena Peña}

Instituto de Estadística de Cataluña (IDESCAT). URL $=<$ http//www.idescat.es/estbasiq/municipi/municipi.stm $>$.

Instituto de Estadística de la Comunidad de Madrid. Base de datos Almudena. $\mathrm{URL}=\langle\mathrm{http} / \mathrm{www} 1$.comadrid.es/destadis/simbad/cgi-bin/simbad.exe $>$.

Instituto Vasco de Estadística (EUSTAT).

$\mathrm{URL}=<$ http://www.eustat.es/spanish/estad/municipal.html >.

Menéndez Bañuelos, B. (1994). La regulación de la estadística en las Comunidades Autónomas. //Boletín de Economía, 7, pp. 13-60.

Orensanz García ; et al. (1995). La difusión estadística. // Cuadernos Aragoneses de Economía, 1, pp. 35-57.

Organización de Cooperación y Desarrollo Económico (1987), La Administración al servicio del público. Madrid: INAP, 1987.

Servicio de Planificación, Estadística y Análisis Económico de la Junta de Extremadura. http:www.junta.ex/consejerias/eic/dgpp/mun2000/home.html > 